K. Ishizaki and N. Yanagihara

Nagoya Math. J.

Vol. 175 (2004), 75-102

\title{
WIMAN-VALIRON METHOD FOR DIFFERENCE EQUATIONS
}

\author{
K. ISHIZAKI AND N. YANAGIHARA
}

\begin{abstract}
Let $f(z)$ be an entire function of order less than $1 / 2$. We consider an analogue of the Wiman-Valiron theory rewriting power series of $f(z)$ into binomial series. As an application, it is shown that if a transcendental entire solution $f(z)$ of a linear difference equation is of order $\chi<1 / 2$, then we have $\log M(r, f)=L r^{\chi}(1+o(1))$ with a constant $L>0$.
\end{abstract}

\section{$\S 1$. Introduction}

In this note we are concerned with an entire solution to a linear difference equation

$$
a_{p}(z) \Delta^{p} f(z)+\cdots+a_{1}(z) \Delta f(z)+a_{0}(z) f(z)=0,
$$

where $\Delta$ is a difference operator defined by $\Delta f(z)=f(z+1)-f(z)$, and $a_{j}(z)=\sum_{k=0}^{A_{j}} a_{k}^{(j)} z^{k}, A_{j}=\operatorname{deg} a_{j}(z)$ and $a_{p}(z) \not \equiv 0$.

Let $f(z)$ be an entire function. We denote by $M(r, f), \mu(r, f)$ and $\nu(r, f)$ the maximum modulus, the maximal term and the central index of $f(z)$, respectively. We use the standard notation of the value distribution theory of entire and meromorphic functions, in particular, Wiman-Valiron theory, see e.g. [5], [8]. Write $\mathfrak{N}_{j}=\left\{(x, y) ; x \geq j, y \leq A_{p-j}-(p-j)\right\}$ for $0 \leq j \leq p$. The Newton polygon for (1.1) is defined as the convex hull of $\mathfrak{N}=\cup_{j=0}^{p} \mathfrak{N}_{j}$.

As is well known, the Wiman-Valiron method is a powerful tool for the study of differential equations, see e.g. [4], [6], [10]. Suppose that $f(z)$ satisfies a linear differential equation

$$
d_{h}(z) f^{(h)}(z)+\cdots+d_{1}(z) f^{\prime}(z)+d_{0}(z) f(z)=0,
$$

Received April 24, 2002.

2000 Mathematics Subject Classification: Primary 39B10, Secondary 30D35 .

Research partially supported by the Grant-in-Aid for General Scientific Research from the Ministry of Education, Science and Culture 11640164 (Toda). 
where $d_{j}(z)=\sum_{k=0}^{D_{j}} d_{k}^{(j)} z^{k}$ and $d_{h}(z) \not \equiv 0$. Then it is well known that

$$
\left\{\begin{aligned}
\log M(r, f) & =L r^{\chi}(1+o(1)), & & \text { as } r \rightarrow \infty \\
\log \mu(r, f) & =L r^{\chi}(1+o(1)), & & \text { as } r \rightarrow \infty \\
\nu(r, f) & =\chi L r^{\chi}(1+o(1)), & & \text { as } r \rightarrow \infty,
\end{aligned}\right.
$$

in which $L$ is a constant, and $\chi$ is a slope of the corresponding Newton polygon for the equation (1.2). We see that $\chi$ is a rational number and gives the order of $f(z)$. Thus $f(z)$ is of finite positive order if transcendental, and is of regular growth. In what follows, for the sake of simplicity " $O(\cdot)$ " and "o(.)" mean " $O(\cdot)$ as $r \rightarrow \infty$ " and " $o(\cdot)$ as $r \rightarrow \infty$ " respectively.

On the other hand, if $f(z)$ satisfies a linear $q$-difference equation

$$
b_{m}(z) f\left(q^{m} z\right)+\cdots+b_{1}(z) f(q z)+b_{0}(z) f(z)=0, \quad 0<|q|<1,
$$

with $b_{j}(z)=\sum_{k=0}^{B_{j}} b_{k}^{(j)} z^{k}$ and $b_{m}(z) \not \equiv 0$, then we have [2],

$$
\left\{\begin{aligned}
\log M(r, f) & =\frac{\sigma}{-2 \log |q|}(\log r)^{2}(1+o(1)) \\
\log \mu(r, f) & =\frac{\sigma}{-2 \log |q|}(\log r)^{2}(1+o(1)) \\
\nu(r, f) & =\frac{\sigma}{-\log |q|} \log r(1+o(1)),
\end{aligned}\right.
$$

where $\sigma$ is a slope of the corresponding Newton polygon for the equation (1.4). This means that $f(z)$ is of order 0 and is also of regular growth.

The natural question arises whether an entire solution $f(z)$ of the linear difference equation (1.1) would be of regular growth in some sense, or not. For instance, the equation $z f(z+1)-f(z)=0$ has an entire solution $f(z)=1 / \Gamma(z)$, which satisfies $m(r, f)=\frac{1}{\pi} r \log r(1+o(1))$, see e.g. [1]. However, the general situation is not so simple. Indeed, for any periodic entire function $\pi(z)$ with period 1 , the product $\pi(z) f(z)$ is also a solution. If $a_{0}(z)=0$, then $f(z)+\pi(z)$ is also a solution. On the other hand, in the case $a_{j}(z)$ are constants, $f(z+\pi(z))$ is also a solution. Hence growth of the solution is not necessarily determined by the equation.

Throughout this note, we mainly pay attention to entire functions of order less than $1 / 2$. We will prove the following theorem.

THEOREM 1.1. Let $f(z)$ be a transcendental entire solution of (1.1) and of order $\chi<1 / 2$. Then we have

$$
\log M(r, f)=L r^{\chi}(1+o(1))
$$


where a rational number $\chi$ is a slope of the Newton polygon for the equation (1.1), and $L>0$ is a constant. In particular, we have $\chi>0$.

We state some remarks below.

Remark 1.1. The Newton polygons for the differential equation (1.2) and the $q$-difference equation (1.4) are given in e.g. [6], [2].

Remark 1.2. For a set $E \in[0, \infty)$, we denote by $\operatorname{lm}(E)$ the logarithmic measure of $E$. Namely $\operatorname{lm}(E)$ stands for the size of the integral $a<b$ being $\int_{a}^{b} d t / t$. Let $\phi(r)$ be a non-decreasing function in $[0, \infty)$, let $\alpha$ be a rational number, and let $C$ be a nonzero constant. Suppose that

$$
\phi(r)=C r^{\alpha}(1+o(1))
$$

holds outside an exceptional set $E \in[0, \infty)$. If $\operatorname{lm}(E)<\infty$, then (1.7) holds for all $r \in[0, \infty)$. See e.g. [6].

Remark 1.3. In general, (1.6) does not always hold for entire solutions of order at least 1 , which is seen by the example $1 / \Gamma(z)$ mentioned above.

Remark 1.4. It is possible to construct an example of a difference equation of the form (1.1) which has two solutions $f$ and $g$ of different order $\chi(f) \neq \chi(g), \chi(f), \chi(g)<1 / 2$. See Example 6.4. It is an open problem to explicitly determine which slopes actually appear as the orders of the entire solutions of (1.1).

We define

$$
z(0)=1, z(1)=z, z(n)=z(z-1) \cdots(z-n+1)=n !\left(\begin{array}{c}
z \\
n
\end{array}\right)(n \geq 2)
$$

We call $\sum_{n=0}^{\infty} a_{n} z(n), a_{n} \in \mathbb{C}, n=0,1, \ldots$, binomial series (or factorial series) in this note. It is easy to see that

$$
\Delta z(n)=n z(n-1), \quad \Delta z(0)=0 \quad \text { and } \quad \Delta z(1)=1
$$

The key idea of the proof of Theorem 1.1 is that we modify the WimanValiron theory for binomial series and apply it to the difference equation.

In Section 2, we study basic properties of binomial series, and we rewrite power series into binomial series. Sections 3 and 4 are devoted to the 
construction of the Wiman-Valiron theory for binomial series. We prove Theorem 1.1 in Section 5. It is not obvious whether there would be a difference equation which has an entire solution of order $<1 / 2$. Finally we give an example of difference equation with entire solution of order less than $1 / 2$.

Acknowledgements. The authors would like to thank the referee for a careful reading of the manuscript and for valuable comments. We also would like to thank Professor Aimo Hinkkanen for his suggestions and encouragements.

\section{§2. Preliminaries}

In this section we state basic properties of the difference operator $\Delta$, and of binomial series.

\subsection{Properties of difference operator}

First we observe $n \in \mathbb{N}$ times iteration of the difference operater to a function $f(z)$. We have

$$
\Delta^{n} f(z)=\sum_{j=0}^{n}\left(\begin{array}{l}
n \\
j
\end{array}\right)(-1)^{n-j} f(z+j)
$$

and

$$
f(z+n)=\sum_{j=0}^{n}\left(\begin{array}{l}
n \\
j
\end{array}\right) \Delta^{j} f(z)
$$

Using the relation (2.1), we can rewrite (1.1) as

$$
\tilde{a}_{p}(z) f(z+p)+\cdots+\tilde{a}_{1}(z) f(z+1)+\tilde{a}_{0}(z) f(z)=0,
$$

where $\tilde{a}_{j}(z), j=0, \ldots, p, \tilde{a}_{p}(z)=a_{p}(z)$ are polynomials.

By induction, we obtain for any $n \leq k$

$$
\Delta^{n} z^{k}=\sum_{\substack{s_{1} \geq 1, \cdots, s_{n} \geq 1 \\ s_{1}+\cdots+s_{n} \leq k}} \frac{k !}{s_{1} ! \cdots s_{n} !\left(k-s_{1}-\cdots-s_{n}\right) !} z^{k-s_{1}-\cdots-s_{n}} .
$$

Next we consider an analogue of Leibniz formula for higher derivatives. For two functions $f(z)$ and $g(z)$, and for $n \geq 1$, we have

$$
\Delta^{n}(f(z) g(z))=\sum_{\ell=0}^{n}\left(\begin{array}{l}
n \\
\ell
\end{array}\right)\left(\sum_{k=0}^{n-\ell}\left(\begin{array}{c}
n-\ell \\
k
\end{array}\right)\left(\Delta^{n-k} f(z)\right)\left(\Delta^{k+\ell} g(z)\right)\right)
$$




\subsection{Properties of binomial series}

Let $f(z)$ be a transcendental entire function of finite order $\chi=\chi(f)<$ $1 / 2$. To construct the the Wiman-Valiron theory for binomial series, we need to prepare the following estimates and calculations. We write

$$
f(z)=\sum_{n=0}^{\infty} b_{n} z^{n}=\sum_{n=0}^{\infty} a_{n} z(n), \quad z(n)=z(z-1) \cdots(z-n+1)
$$

where $a_{n}, b_{n} \in \mathbb{C}$. We define

$$
f^{\#}(z)=\sum_{n=0}^{\infty}\left|b_{n}\right| z^{n}
$$

Obviously $\chi\left(f^{\#}\right)=\chi(f)$, and $M\left(r, f^{\#}\right)=f^{\#}(r) \geq M(r, f)$. Write

$$
f^{\#}(z)=\sum_{n=0}^{\infty} a_{n}^{\#} z(n)
$$

We write $\left.\Delta^{n} z^{k}\right|_{z=0}=\Lambda_{n, k}$. It follows from (2.6), (2.7) and (2.3) that

$$
\begin{aligned}
& a_{n}=\frac{1}{n !} \sum_{k=n}^{\infty} \Lambda_{n, k} b_{k}, \quad a_{n}^{\#}=\frac{1}{n !} \sum_{k=n}^{\infty} \Lambda_{n, k}\left|b_{k}\right|, \\
& \Lambda_{n, k}=\sum_{\substack{s_{1} \geq 1, \cdots, s_{n} \geq 1 \\
s_{1}+\cdots+s_{n}=k}} \frac{k !}{s_{1} ! \cdots s_{n} !} .
\end{aligned}
$$

We see that

$$
n^{k} \geq \Lambda_{n, k}
$$

Obviously $a_{n}^{\#} \geq 0$. Recall that $\chi$ is represented by the Taylor coefficients as, see e.g. [3, p. 9],

$$
\chi=\limsup _{k \rightarrow \infty} \frac{k \log k}{\log \frac{1}{\left|b_{k}\right|} .}
$$

We fix a sufficiently small $\epsilon>0$ satisfying $1 /(\chi+\epsilon)>2$, and for the sake of simplicity we put $\gamma=1 /(\chi+\epsilon)$. Then we see that $\left|b_{k}\right| \leq k^{-\gamma k}$ for sufficiently 
large $k$. We get by Stirling's formula, for some constants $K_{0}$ and $K_{1}>0$,

$$
\begin{aligned}
\left|a_{n}\right| \leq a_{n}^{\#} & \leq K_{0} \frac{e^{n}}{n^{n}} \sum_{k=n}^{\infty} \frac{n^{k}}{k^{\gamma k}} \leq K_{0} \frac{e^{n}}{n^{\gamma n}} \sum_{k=n}^{\infty} \frac{n^{k}}{n^{n}} \frac{n^{\gamma n}}{k^{\gamma k}} \\
& =K_{0} \frac{e^{n}}{n^{\gamma n}} \sum_{k=n}^{\infty}\left(\frac{n}{k^{\gamma}}\right)^{k-n}\left(\frac{n}{k}\right)^{\gamma n} \\
& \leq K_{0} \frac{e^{n}}{n^{\gamma n}} \sum_{k=n}^{\infty} \frac{1}{k^{(\gamma-1)(k-n)}} \leq K_{1} \frac{e^{n}}{n^{\gamma n}}
\end{aligned}
$$

if $n$ is sufficiently large. Retaking $K_{1}$, if necessary, we may suppose that this is valid for $n \geq 1$. Hence in the domain $|z|<r$ we have for $K_{1}$ and $K_{2}(r)$ which are independent of $N$

$$
\begin{aligned}
& \sum_{n=1}^{N}\left|a_{n}^{\#} z(n)\right| \leq \sum_{n=1}^{N}\left|a_{n}^{\#}\right||r(r+1) \cdots(r+n-1)| \\
\leq & K_{1} \sum_{n=1}^{N} \frac{e^{n}}{n^{\gamma n}}([r]+n) ! \leq K_{2}(r) \sum_{n=1}^{N}\left(\frac{4 n^{2}}{n^{\gamma}}\right)^{n} \sqrt{n}
\end{aligned}
$$

where $[r]$ is the greatest integer not exceeding $r$. This implies that the right-hand side of (2.7) converges uniformly on every compact subset in $\mathbb{C}$.

We define

$$
z^{*(k)}=z(z+1) \cdots(z+k-1), z^{*(0)}=1, z^{*(1)}=z,
$$

and, for $a_{n}^{\#}$ given by (2.8), we put

$$
f^{*}(z)=\sum_{n=0}^{\infty} a_{n}^{\#} z^{*(n)}=\sum_{n=0}^{\infty} a_{n}^{\#} z(z+1) \cdots(z+n-1)=\sum_{n=0}^{\infty} b_{n}^{*} z^{n}
$$

Similarly to the proof of the convergence of $\sum_{n=0}^{\infty} a_{n}^{\#} z(n)$, we see that $\sum_{n=0}^{\infty} a_{n}^{\#} z^{*(n)}$ converges uniformly on every compact subset in $\mathbb{C}$. Next we estimate $b_{n}^{*}$ in $(2.11)$. Write $z(k)=z^{k}+\sum_{j=1}^{k-1} \eta_{j}^{(k)} z^{j}$. Then we have $z^{*(k)}=z^{k}+\sum_{j=1}^{k-1}\left|\eta_{j}^{(k)}\right| z^{j}$ and

$$
\left|\eta_{j}^{(k)}\right| \leq j(j+1) \cdots(k-1) \frac{(k-1) !}{(j-1) !(k-j) !}=\left(\frac{(k-1) !}{(j-1) !}\right)^{2} \frac{1}{(k-j) !}
$$


We get by (2.11), with a constant $K_{2}>0$,

$$
\begin{aligned}
b_{n}^{*} & =\left.\frac{1}{n !} \sum_{k=n}^{\infty} a_{k}^{\#} \frac{d^{n}}{d z^{n}} z^{*(k)}\right|_{z=0}=\sum_{k=n}^{\infty} a_{k}^{\#}\left|\eta_{n}^{(k)}\right| \\
& \leq K_{1} \sum_{k=n}^{\infty} \frac{e^{k}}{k^{\gamma k}}\left(\frac{(k-1) !}{(n-1) !}\right)^{2} \frac{1}{(k-n) !} \\
& \leq \frac{K_{1} n^{2}}{(n !)^{2}} \sum_{k=n}^{\infty} \frac{e^{-k}}{k^{(\gamma-2) k}} \frac{1}{(k-n) !} \leq \frac{K_{1} n^{2}}{(n !)^{2}} \sum_{k=n}^{\infty} \frac{e^{-k}}{n^{(\gamma-2) k}} \leq K_{2} \frac{n^{2} e^{n}}{n^{\gamma n}}
\end{aligned}
$$

where $\eta_{n}^{(n)}=1$. Hence we see that $f^{*}(z)$ is an entire function having the Taylor expansion as in the right-hand side of (2.11). Further (2.13) shows $\chi\left(f^{*}\right) \leq 1 / \gamma=\chi(f)+\epsilon$, and hence $\chi\left(f^{*}\right) \leq \chi(f)$. We have $\chi\left(f^{*}\right)=\chi(f)$ since $M(r, f) \leq M\left(r, f^{*}\right)$ as easily seen. Obviously $\mu(r, f)=\mu\left(r, f^{\#}\right)$ and $\nu(r, f)=\nu\left(r, f^{\#}\right)$. We write

$$
\mu^{\circledast}(r)=\mu^{\circledast}\left(r, f^{\#}\right)=\mu^{\circledast}\left(r, f^{*}\right)=\sup _{0 \leq n<\infty}\left(\sup _{|z|=r}\left|a_{n}^{\#} z(n)\right|\right),
$$

Note that $\mu^{\circledast}(r)=\left|a_{N}^{\#}\right| r^{*(N)}=\left|a_{N}^{\#}\right| r(r+1) \cdots(r+N-1)$ for some $N$. We define

$$
\nu^{\circledast}(r)=\nu^{\circledast}\left(r, f^{\#}\right)=\nu^{\circledast}\left(r, f^{*}\right)=\max \left\{n|| a_{n}^{\#} \mid r^{*(n)}=\mu^{\circledast}(r)\right\} .
$$

We call $\mu^{\circledast}(r)$ and $\nu^{\circledast}(r)$ the $\circledast$-maximal term and the $\circledast$-central index of $f^{*}(z)$ (or call sometimes of $f^{\#}(z)$ ), receptively. We have $\chi(f)=\chi\left(f^{\#}\right)=$ $\chi\left(f^{*}\right)$. Since $\mu^{\circledast}(r) \leq M\left(r, f^{*}\right)$, we have

$$
\chi\left(\mu^{\circledast}\right)=\limsup _{r \rightarrow \infty} \frac{\log \log \mu^{\circledast}(r)}{\log r} \leq \chi\left(f^{*}\right)<\frac{1}{2} .
$$

In the case $R>r$, we have $(R+\ell) /(r+\ell)<R / r$, for any $\ell>0$. Hence writing $\nu^{\circledast}(r)$ as $N(r)$, we have for $N=N(r)$,

$$
\begin{aligned}
\left(\frac{R+N-1}{r+N-1}\right)^{N} & \leq\left(\frac{R}{r}\right)\left(\frac{R+1}{r+1}\right) \cdots\left(\frac{R+N-1}{r+N-1}\right) \\
& =\frac{a_{N}^{\#} R^{*(N)}}{a_{N}^{\#} r^{*(N)}} \leq \frac{\mu^{\circledast}(R)}{\mu^{\circledast}(r)}, \\
N \log \left(\frac{R+N-1}{r+N-1}\right) & \leq \log \mu^{\circledast}(R)-\log \mu^{\circledast}(r) \leq \log \mu^{\circledast}(R),
\end{aligned}
$$


provided that $r$ is sufficiently large. Set $R=2 r+(N-1)$. Then $N(r) \log 2 \leq$ $\log \mu^{\circledast}(2 r+N(r)-1)$ and

$$
\begin{aligned}
& \frac{\log N(r)}{\log r}+\frac{\log \log 2}{\log r} \\
& \quad \leq \frac{\log \log \mu^{\circledast}(2 r+N(r)-1)}{\log (2 r+N(r)-1)} \frac{\log (2 r+N(r)-1)}{\log r} .
\end{aligned}
$$

Put $A=\chi\left(\mu^{\circledast}\right)+\epsilon<1 / 2$. If we assume there is a sequence $r_{k} \uparrow \infty$ such that $N\left(r_{k}\right)>r_{k}$. Then we have $2 r_{k}-1 \leq 2 N\left(r_{k}\right)$ and from (2.17)

$$
\begin{aligned}
& 1 \leq \frac{\log N\left(r_{k}\right)}{\log r_{k}} \leq A \frac{\log N\left(r_{k}\right)+\log 3}{\log r_{k}}-\frac{\log \log 2}{\log r_{k}}, \\
& (1-A)<(1-A) \frac{\log N\left(r_{k}\right)}{\log r_{k}}<\frac{A \log 3-\log 2}{\log r_{k}}
\end{aligned}
$$

if $k$ is large. Letting $k \rightarrow \infty$, we get a contradiction, since we now suppose $A<1$. Hence $N(r) \leq r$ for any $r$ sufficiently large. Hence it follows from (2.17) that

$$
\frac{\log N(r)}{\log r} \leq A \frac{\log (3 r-1)}{\log r}-\frac{\log \log 2}{\log r},
$$

for any $r$ sufficiently large. Therefore, we obtain with $\gamma=1 /\left(\chi\left(\mu^{\circledast}\right)+\epsilon\right)>2$,

$$
\limsup _{r \rightarrow \infty} \frac{\log \nu^{\circledast}(r)}{\log r} \leq \chi\left(\mu^{\circledast}\right) \quad \text { and } \quad \nu^{\circledast}(r)^{\gamma} \leq r .
$$

On the other hand, since $\log a_{N}^{\#} \leq 0$, if $N=N(r)$ is sufficiently large, we have

$$
\begin{aligned}
\log \mu^{\circledast}(r) & =\log a_{N}^{\#}+N \log r+\sum_{j=1}^{N-1} \log \left(1+\frac{j}{r}\right) \\
& \leq N \log r+\sum_{j=1}^{N-1} \log \left(1+j^{-\gamma+1}\right)=N \log r+O(1)
\end{aligned}
$$

which implies that

$$
\chi\left(\mu^{\circledast}\right) \leq \limsup _{r \rightarrow \infty} \frac{\log \nu^{\circledast}(r)}{\log r} .
$$


By $(2.8)$ we see $\left|b_{n}\right| \leq a_{n}^{\#}$ hence $\mu(r) \leq \mu^{\circledast}(r)$. Thus, if $\chi(f)<1 / 2$, then, by $(2.15)$,

$$
\begin{aligned}
\chi(f) & =\chi\left(f^{*}\right)=\chi(\mu)=\chi\left(\mu^{\circledast}\right) \\
& =\limsup _{r \rightarrow \infty} \frac{\log \nu(r, f)}{\log r}=\limsup _{r \rightarrow \infty} \frac{\log \nu^{\circledast}(r)}{\log r}
\end{aligned}
$$

We now give definitions of maximal term and central index for the binomial series (2.5). We write

$$
\begin{aligned}
& \mu^{*}(r, f)=\sup _{0 \leq n<\infty}\left(\sup _{|z|=r}\left|a_{n} z(n)\right|\right) \\
& \nu^{*}(r, f)=\max \left\{n|| a_{n} \mid r^{*(n)}=\mu^{*}(r)\right\} .
\end{aligned}
$$

and call them the $*$-maximal term of $f(z)$, and the $*$-central index respectively.

Remark 2.1. Obviously $\mu^{*}(r) \leq \mu^{\circledast}(r)$. Using just the same arguments as above, we see that $\chi\left(\mu^{*}\right) \leq \chi\left(\mu^{\circledast}\right)<1 / 2$ and $\nu^{*}(r)^{\gamma}<r$.

Remark 2.2. When $r>n$ we have for the binomial series in (2.5),

$$
\begin{aligned}
a_{n} & =\frac{1}{2 \pi i} \int_{|\zeta|=r} \frac{f(\zeta)}{\zeta(n+1)} d \zeta \\
& =\frac{1}{2 \pi i} \int_{|\zeta|=r} \frac{f(\zeta)}{\zeta(\zeta-1) \cdots(\zeta-n+1)(\zeta-n)} d \zeta
\end{aligned}
$$

which corresponds to the Cauchy integral formula. In fact, we can write

$$
\begin{aligned}
\frac{f(\zeta)}{\zeta(n+1)} & =\sum_{\ell=0}^{\infty} \frac{a_{\ell} \zeta(\ell)}{\zeta(n+1)}=\sum_{\ell=0}^{n-1} \frac{a_{\ell}}{(\zeta-\ell) \ldots(\zeta-n)}+\frac{a_{n}}{\zeta-n}+\varphi(\zeta) \\
& =\sum_{\ell=0}^{n-1} \sum_{k=0}^{n-\ell} \frac{B_{\ell k}}{\zeta-\ell-k}+\frac{a_{n}}{\zeta-n}+\varphi(\zeta),
\end{aligned}
$$

where $\varphi(\zeta)$ is an analytic function, and $B_{\ell k}$ are constants satisfying $\sum_{k=0}^{n-\ell} B_{\ell k}=0$ for all $\ell$. This gives (2.21) immediately.

Suppose $r \geq n^{\gamma}, \gamma>2$. Then

$$
\left|a_{n}\right| r^{*(n)} \leq \frac{r(r+1) \cdots(r+n-1)}{(r-1)(r-2) \cdots(r-n)} M(r, f) \leq K_{n} M(r, f),
$$


where

$$
K_{n} \leq\left(1+\frac{1}{n^{\gamma-1}}\right)^{n}\left(1-\frac{1}{n^{\gamma-1}}\right)^{-n}<1+\epsilon_{n}, \quad \epsilon_{n} \rightarrow 0 \quad \text { as } n \rightarrow \infty .
$$

Remark 2.3. It is seen that the *-central index $\nu^{*}(r)$ is a increasing function in $r$. In fact, we fix $r$ arbitrary, and put $\nu^{*}(r)=N$ for the sake of brevity. For any $\rho>r$, we have $\left|a_{N}\right| r^{*(N)} \leq\left|a_{N}\right| \rho^{*(N)} \leq\left|a_{M}\right| \rho^{*(M)}$, where $M=\nu^{*}(\rho)$. Assume that $M<N$. From the inequalties above, we have $\left|a_{M}\right| /\left|a_{N}\right| \geq(\rho+M) \ldots(\rho+N-1)$. On the other hand, by definition we have $\left|a_{N}\right| r^{*(N)} \geq\left|a_{M}\right| r^{*(M)}$, namely $\left|a_{M}\right| /\left|a_{N}\right| \leq(r+M) \ldots(r+N-1)$. Combining these estimates, we have $(r+M) \ldots(r+N-1) \geq(\rho+M) \ldots(\rho+$ $N-1)$, a contradiction. Hence we see that $M \geq N$.

\section{§3. A modification of the Wiman-Valiron theory}

Let $f(z)$ and $f^{*}(z)$ be entire functions which are represented as in (2.5) and (2.11). We construct a modified Wiman-Valiron theory for $f(z)$ and $f^{*}(z)$ following the idea in [5], [7]. Put

$$
\begin{aligned}
& \alpha^{\prime}(t)=\left\{\begin{array}{l}
-\frac{1}{t \log t(\log \log t)^{1+\delta}}, \quad t \geq t_{0} \\
\alpha^{\prime}\left(t_{0}\right), \quad 0 \leq t \leq t_{0},
\end{array}\right. \\
& \alpha(t)=\int_{0}^{t} \alpha^{\prime}(\tau) d \tau=\alpha^{\prime}\left(t_{0}\right) t_{0}-\frac{1}{\delta} \frac{1}{\left(\log \log t_{0}\right)^{\delta}}+\frac{1}{\delta} \frac{1}{(\log \log t)^{\delta}},
\end{aligned}
$$

in which $\delta>0$ is a positive number, $t_{0} \geq e^{e}$. Then $\alpha(t) \in C^{1}[0, \infty)$ and $-\log 2 \leq \alpha(t) \leq 0$ if $t_{0}$ is sufficiently large. Write

$$
\alpha_{n}=\exp \left[\int_{0}^{n} \alpha(t) d t\right], \quad \rho_{n}=\exp [-\alpha(n)]
$$

Then

$$
1<\rho_{0}<\frac{\alpha_{0}}{\alpha_{1}}, \quad \frac{\alpha_{n-1}}{\alpha_{n}}<\rho_{n}<\frac{\alpha_{n}}{\alpha_{n+1}}, \quad n \geq 1
$$

so that $\rho_{n}$ increases. For $f(z)$, a value $r$ is said to be $*$-normal if for some $N$

$$
\left\{\begin{array}{l}
\left|a_{n}\right| r^{*(n)} \leq\left|a_{N}\right| r^{*(N)} \frac{\alpha_{n} \rho_{N}^{n}}{\alpha_{N} \rho_{N}^{N}} \quad(n \geq N) \\
\left|a_{n}\right| r^{*(n)} \leq\left|a_{N}\right| r^{*(N)}\left(1+\epsilon_{n, N}\right) \frac{\alpha_{n} \rho_{N}^{n}}{\alpha_{N} \rho_{N}^{N}} \quad(n<N),
\end{array}\right.
$$


in which

$$
\epsilon_{n, N}=\frac{n}{N^{\gamma}}+\frac{n+1}{N^{\gamma}}+\cdots+\frac{N-1}{N^{\gamma}}<\frac{1}{2} \frac{1}{N^{\gamma-2}} \quad(n<N),
$$

where $\gamma=1 /(\chi(f)+\epsilon)>2$. We have for $k=|N-n|$,

$$
\begin{aligned}
\frac{\alpha_{n} \rho_{N}^{n}}{\alpha_{N} \rho_{N}^{N}} & =\exp \left[\int_{N}^{n}(\alpha(t)-\alpha(N)) d t\right] \leq \exp \left[\int_{N}^{n}(t-N) \alpha^{\prime}(t) d t\right] \\
& \leq \exp \left[-\frac{1}{2} \alpha_{N, k} k^{2}\right], \quad \alpha_{N, k}=\min _{|t-N| \leq k}\left|\alpha^{\prime}(t)\right| .
\end{aligned}
$$

In particular, in the case $n<N, N \geq t_{0}$, we have

$$
\begin{aligned}
\frac{\alpha_{n} \rho_{N}^{n}}{\alpha_{N} \rho_{N}^{N}} & \leq \exp \left[-\frac{1}{2} \alpha_{N, k}^{(-)} k^{2}\right], \\
\alpha_{N, k}^{(-)} & =\min _{N-k \leq t \leq N}\left|\alpha^{\prime}(t)\right|=\frac{1}{N \log N(\log \log N)^{1+\delta}} .
\end{aligned}
$$

Thus $\left(1+\epsilon_{n, N}\right) \alpha_{n} \rho_{N}^{n} / \alpha_{N} \rho_{N}^{N}<1$ for $n<N$. Hence if $r$ is $*$-normal, then

$$
\left|a_{n}\right| r^{*(n)}=\left|a_{n}\right| r(r+1) \cdots(r+n-1)<\left|a_{N}\right| r^{*(N)}
$$

for $n \neq N$, and $N$ is necessarily the $*$-central index in the sense (2.19).

THEOREM 3.1. Suppose $f(z)$ is an entire function of order $\chi<1 / 2$. If $\rho_{n}, \alpha_{n}$ are defined as above, then the set $E$ of $*$-exceptional (that is, not *-normal) $r$ has finite logarithmic measure.

Proof. If the *-central index $N(r)$ jumps from $N$ to $N+k$ as $r$ increases through the value $r_{N+1}$, we define $r_{N+1}=\cdots=r_{N+k}$. We see

$$
\begin{gathered}
\left|a_{N+k}\right| r_{N+k}\left(r_{N+k}+1\right) \cdots\left(r_{N+k}+N+k-1\right) \\
=\left|a_{N}\right| r_{N+1}\left(r_{N+1}+1\right) \cdots\left(r_{N+1}+N-1\right), \\
\left|a_{N+k}\right|=\frac{\left|a_{N}\right|}{\left(r_{N+k}+N\right) \cdots\left(r_{N+k}+N+k-1\right)} \\
=\frac{\left|a_{N}\right|}{\left(r_{N+1}+N\right) \cdots\left(r_{N+k}+N+k-1\right)}, \\
\left|a_{N+\nu}\right| \leq \frac{\left|a_{N}\right|}{\left(r_{N+1}+N\right) \cdots\left(r_{N+\nu}+N+\nu-1\right)} \quad(1 \leq \nu \leq k) .
\end{gathered}
$$


Thus we always have

$$
\frac{\left|a_{N}\right|}{\left|a_{0}\right|} \leq \frac{1}{r_{1}\left(r_{2}+1\right) \cdots\left(r_{N}+N-1\right)}
$$

On the other hand we have

$$
\begin{aligned}
\frac{\alpha_{N}}{\alpha_{0}} & \geq \frac{1}{\rho_{1} \cdots \rho_{N}} \\
\frac{\left|a_{N}\right|}{\alpha_{N}} & \leq \frac{\left|a_{0}\right|}{\alpha_{0}} \frac{\rho_{1}}{r_{1}} \frac{\rho_{2}}{r_{2}+1} \frac{\rho_{3}}{r_{3}+2} \cdots \frac{\rho_{N}}{r_{N}+N-1} .
\end{aligned}
$$

Since $r_{N}>N^{\gamma}, \gamma>2$, if $r_{N}$ is sufficiently large (cf. Remark 2.1), and $\rho_{n} \leq 2$, we have

$$
A_{N}=\frac{\left|a_{N}\right|}{\alpha_{N}} \leq \frac{C_{0} 2^{N}}{(N !)^{\gamma}} \leq \frac{C_{0}\left(2 e^{\gamma}\right)^{N}}{N^{\gamma N}}
$$

where $C_{0}$ is some positive number independent of $N$. Thus $F(z)=$ $\sum_{n=0}^{\infty} A_{n} z(n)=\sum_{n=0}^{\infty} A_{n} z(z-1) \cdots(z-n+1)$ is an entire function (cf. the proof of convergence of (2.7)), which is of order $\leq 1 / \gamma<1 / 2$ as seen by (2.13). It follows that, for $0<\rho<\infty, F(z)$ has a $*$-maximal term for $|z|=\rho$. If $M=M(\rho)$ is the $*$-central index of $F(z)$ for $|z|=\rho$, we deduce $A_{n} \rho^{*(n)} \leq A_{M} \rho^{*(M)}$, hence for $n>M$,

$$
\begin{aligned}
& \frac{\left|a_{n}\right| \rho^{*(n)} \rho_{M}^{n-M}}{\left|a_{M}\right| \rho^{*(M)}}=\frac{\left|a_{n}\right|(\rho+M) \cdots(\rho+n-1) \rho_{M}^{n-M}}{\left|a_{M}\right|} \leq \frac{\alpha_{n} \rho_{M}^{n}}{\alpha_{M} \rho_{M}^{M}}, \quad \text { and } \\
& \frac{\left|a_{n}\right|\left(\rho \rho_{M}\right)^{*(n)}}{\left|a_{M}\right|\left(\rho \rho_{M}\right)^{*(M)}}=\frac{\left|a_{n}\right|\left(\rho \rho_{M}+M\right) \cdots\left(\rho \rho_{M}+n-1\right)}{\left|a_{M}\right|} \\
& \leq \frac{\left|a_{n}\right|\left(\rho \rho_{M}+\rho_{M} M\right) \cdots\left(\rho \rho_{M}+\rho_{M}(n-1)\right)}{\left|a_{M}\right|}=\frac{\left|a_{n}\right| \rho^{*(n)} \rho_{M}^{n-M}}{\left|a_{M}\right| \rho^{*(M)}} \leq \frac{\alpha_{n} \rho_{M}^{n}}{\alpha_{M} \rho_{M}^{M}} .
\end{aligned}
$$

Note that for $0<x_{j}<1, \sum_{j=1}^{n} x_{j}<1$ we have $\prod_{j=1}^{n}\left(1+x_{j} / 2\right) \leq 1+$ 
$\sum_{j=1}^{n} x_{j}$. For $n<M$, since $\rho \geq M^{\gamma}$,

$$
\begin{aligned}
& \frac{\left|a_{n}\right|\left(\rho \rho_{M}\right)^{*(n)}}{\left|a_{M}\right|\left(\rho \rho_{M}\right)^{*(M)}}=\frac{\left|a_{n}\right|}{\left|a_{M}\right|\left(\rho \rho_{M}+n\right) \cdots\left(\rho \rho_{M}+M-1\right)} \\
& =\frac{\left|a_{n}\right| /\left|a_{M}\right|}{\left(\rho \rho_{M}+\rho_{M} n\right) \cdots\left(\rho \rho_{M}+\rho_{M}(M-1)\right)} \\
& =\frac{\mid\left(\rho \rho_{M}+\rho_{M} n\right) \cdots\left(\rho \rho_{M}+\rho_{M}(M-1)\right)}{\left(\rho \rho_{M}+n\right) \cdots\left(\rho \rho_{M}+(M-1)\right)} \\
& \leq \frac{\left|a_{n}\right| \rho^{*(n)} \mid \rho^{*(n)}}{\left|a_{M}\right| \rho^{*(M)} \rho_{M}^{M-n}} \frac{(1+n / \rho) \cdots(1+(M-1) / \rho)}{\left(1+n /\left(\rho \rho_{M}\right)\right) \cdots\left(1+(M-1) /\left(\rho \rho_{M}\right)\right)} \\
& \leq \frac{\left|a_{n}\right| \rho^{*(n)} \rho_{M}^{n}}{\left|a_{M}\right| \rho^{*(M)} \rho_{M}^{M}} \prod_{\ell=n}^{M-1}(1+n / \rho) \cdots(1+(M-1) / \rho) \\
& \leq \frac{\left|a_{n}\right| \rho^{*(n)}}{\left|a_{M}\right| \rho^{*(M)}} \cdot \frac{\rho_{M}^{n}}{\rho_{M}^{M}} \cdot\left(1+\epsilon_{n, M}\right) \leq\left(1+\epsilon_{n, M}\right) \frac{\mid a_{n} \rho_{M}^{n}}{\alpha_{M} \rho_{M}^{M}} .
\end{aligned}
$$

Thus, if $r=\rho \rho_{M}$, it follows that $N(r)=M$, and the inequality (3.2) holds. Hence $r$ is $*$-normal if $r$ is of this form, i.e. if we can write $r=\rho \rho_{M}$ where $M$ is the $*$-central index of $F(z)$ for $|z|=\rho$.

Let $\left(R_{n}, R_{n+1}\right)$ be the interval in which the $*$-central index of $F(z)$ is $n$. Then the intervals $\left(R_{n} \rho_{n}, R_{n+1} \rho_{n}\right)$ are $*$-normal for $n$ of $f(z)$, so that the $*$-exceptional values of $r$ are contained in the complementary intervals of the form $\left(R_{n} \rho_{n-1}, R_{n} \rho_{n}\right)$. Let $E_{N}$ be the union of these complementary intervals for $n=1, \ldots, N$. Then

$$
\operatorname{lm}\left(E_{N}\right) \leq \sum_{n=1}^{N} \int_{R_{n} \rho_{n-1}}^{R_{n} \rho_{n}} \frac{d t}{t}=\log \frac{\rho_{N}}{\rho_{0}} .
$$

Also the *-exceptional values satisfying $r \leq R_{N+1} \rho_{N}$ are contained in $E_{N}$.

We recall that the *-central index of $f(z)$ is $n$ in the interval $\left(R_{n} \rho_{n}, R_{n+1} \rho_{n}\right)$, so that $r_{n} \leq \rho_{n} R_{n}$. Thus if $r_{n} \leq r \leq r_{n+1}$, we deduce that $r \leq \rho_{n+1} R_{n+2}$, so that the $*$-exceptional values in $(0, r)$ are contained in $E_{n+1}$ and so have logarithmic measure at most $\log \left(\rho_{n+1} / \rho_{0}\right)$. Thus if $E$ is the set of $*$-exceptional values, we have

$$
\operatorname{lm}(E \cap[1, r]) \leq \log \left(\rho_{n+1} / \rho_{0}\right), \quad r_{n} \leq r \leq r_{n+1} .
$$

Since $\rho_{N}$ is bounded above, so is $\operatorname{lm}(E \cap[1, r])$ as $r \rightarrow \infty$. 
By Theorem 3.1, and (3.2), (3.3) and (3.4), we obtain the following theorem.

TheOREm 3.2. Let $f(z)$ be as in (2.5). Let $E$ be the $*$-exceptional set of $r$. Then $\operatorname{lm}(E)<\infty$. For $r \notin E$ and $N=\nu^{*}(r)$ we have

$$
\left\{\begin{array}{l}
\frac{\left|a_{N+k}\right| r^{*(N+k)}}{\mu^{*}(r)} \leq \exp \left(-\frac{1}{2} b(k+N) k^{2}\right), \quad k \in \mathbb{N}, \\
\frac{\left|a_{N-k}\right| r^{*(N-k)}}{\mu^{*}(r)} \leq\left(1+\epsilon_{N}\right) \exp \left(-\frac{1}{2} b(N) k^{2}\right), \quad 0 \leq k<N,
\end{array}\right.
$$

where $\epsilon_{N}=1 /\left(2 N^{\gamma-2}\right)$ and

$$
b(N)=1 /\left(N \log N(\log \log N)^{1+\delta}\right) .
$$

Furthermore, we obtain the following theorem.

Theorem 3.3. Let $b(N)$ be as in (3.6). Let $\omega>0$ be a constant. Write

$$
k=\left[\left\{\frac{\omega}{b(N)} \log \frac{1}{b(N)}\right\}^{1 / 2}\right]
$$

$([x]$ is the greatest integer not exceeding $x \in \mathbb{R})$.

Then for any fixed real $h$ and $\omega_{1}, 0<\omega_{1}<\omega$ we have

$$
\sum_{|n-N| \geq k} n^{h}\left|a_{n}\right| r^{*(n)}=o\left(\mu^{*}(r) N^{h} b(N)^{(1 / 2) \omega_{1}-(1 / 2)}\right)
$$

uniformly as $r \rightarrow \infty, r \notin E$.

This theorem can be proved similarly to Lemma 2 in [5, p. 326].

Proof. Let $\eta$ be an arbitrary number satisfying $0<\eta \leq 1 / 2$. In the proof, we divide the left-hand side of (3.8) into four parts:

$$
\begin{aligned}
& \sum_{n \leq N \mid \geq k} n^{h}\left|a_{n}\right| r^{*(n)} \\
= & \sum_{n \leq(1-\eta) N} n^{h}\left|a_{n}\right| r^{*(n)}+\sum_{N+k \leq n<(1+\eta) N} \sum_{\substack{(1-\eta) N<n \leq N-k \\
n^{h}\left|a_{n}\right| r^{*(n)}}} n^{h}\left|a_{n}\right| r^{*(n)} \\
& +\sum_{(1+\eta) N \leq n} n^{h}\left|a_{n}\right| r^{*(n)} .
\end{aligned}
$$


First we consider the case $(1+\eta) N \leq n$. Since $r$ is $*$-normal, we have for $n=N+p, p \in \mathbb{N}$

$$
\frac{n^{h}\left|a_{n}\right| r^{*(n)}}{\left|a_{N}\right| r^{*(N)}} \leq \frac{n^{h} \alpha_{n} \rho_{N}^{n}}{\alpha_{N} \rho_{N}^{N}} \leq \exp \left(-\frac{1}{2} \alpha_{N, p} p^{2}+h \log (N+p)\right),
$$

where $\alpha_{N, p}$ is defined in (3.3). In this case, we see that $\alpha_{N, p}=1 /[(N+$ $\left.p) \log (N+p)(\log \log (N+p))^{1+\delta}\right]$. Since $\nu^{*}(r)=N$ tends to infinity (as $r \rightarrow \infty)$, we choose $r_{0}$ so large that the following inequality holds for $p \geq \eta N$ and for $r \geq r_{0}$

$$
\begin{aligned}
-\frac{1}{2} \alpha_{N, p} p^{2}+h \log (N+p) \leq & -\frac{\eta}{2(1+\eta)} \frac{p}{\log (N+p)(\log \log (N+p))^{1+\delta}} \\
& +h \log p+h \log \left(1+\frac{1}{\eta}\right) \\
\leq & -p^{1 / 2} .
\end{aligned}
$$

Hence we have

$$
\frac{1}{\left|a_{N}\right| r^{*(N)}} \sum_{(1+\eta) N \leq n} n^{h}\left|a_{n}\right| r^{*(n)} \leq \sum_{\eta N \leq p} e^{-p^{1 / 2}} \leq \int_{\eta N-1}^{\infty} e^{-x^{1 / 2}} d x .
$$

For $x \geq \eta N-1$, we have $x^{1 / 2} \geq \frac{1}{2} x^{1 / 2}+\frac{1}{2}(\eta N)^{1 / 2}-1$, which implies that

$$
\int_{\eta N-1}^{\infty} e^{-x^{1 / 2}} d x \leq e^{-\frac{1}{2}(\eta N)^{1 / 2}} \int_{0}^{\infty} e^{1-\frac{1}{2} x^{1 / 2}}
$$

Hence we see that

$$
\frac{1}{\left|a_{N}\right| r^{*(N)}} \sum_{(1+\eta) N \leq n} n^{h}\left|a_{n}\right| r^{*(n)}=O\left(e^{-(\eta N)^{1 / 2} / 2}\right) .
$$

Secondly we consider the case $n \leq(1-\eta) N$. Similarly to the case above, for $n=N-p, p \in \mathbb{N}$

$$
\frac{n^{h}\left|a_{n}\right| r^{*(n)}}{\left|a_{N}\right| r^{*(N)}} \leq\left(1+\epsilon_{n, N}\right) \exp \left(-\frac{1}{2} \alpha_{N, p}^{(-)} p^{2}+h \log (N-p)\right),
$$

where $\alpha_{N, p}^{(-)}$is defined in (3.4). This gives that

$$
\frac{1}{\left|a_{N}\right| r^{*(N)}} \sum_{n \leq(1-\eta) N} n^{h}\left|a_{n}\right| r^{*(n)}=O\left(e^{-(\eta N)^{1 / 2} / 2}\right) .
$$


Finally we treat the case $(1-\eta) N<n \leq N-k$ and $N+k \leq n<$ $(1+\eta) N$. Given $\epsilon>0$ satisfying $\epsilon<\epsilon_{N}$ (cf (3.5)), we choose $\eta$ so small that

$$
b(N+|p|)>(1-\epsilon) b(N) \quad \text { for } \quad|p| \leq \eta N, \quad \text { and } \quad(1-\eta)^{-|h|}<1+\epsilon,
$$

which is possible under the hypotheses of Theorem 3.3. Then (3.5) yields for $n=N+p,|p|<\eta N$,

$$
\frac{n^{h}\left|a_{n}\right| r^{*(n)}}{\left|a_{N}\right| r^{*(N)}} \leq\left(1+\epsilon_{N}\right)^{2} N^{h} e^{-b^{*} p^{2}}
$$

where $b^{*}=\frac{1}{2}(1-\epsilon) b(N)$.

It follows from (3.10), (3.10), (3.11) and (3.12) that

$$
\begin{aligned}
& \sum_{|n-N| \geq k} n^{h}\left|a_{n}\right| r^{*(n)} \\
& \quad \leq 2\left(1+\epsilon_{N}\right)^{2} N^{h} \mu^{*}(r)\left(\sum_{p=k}^{\infty} e^{-b^{*} p^{2}}+O\left(e^{-(\eta N)^{1 / 2} / 2}\right)\right) .
\end{aligned}
$$

We have

$$
\begin{aligned}
\sum_{p=k}^{\infty} e^{-b^{*} p^{2}} & \leq \int_{k-1}^{\infty} e^{-b^{*} x^{2}} d x=\left(b^{*}\right)^{-1 / 2} \int_{y_{0}}^{\infty} e^{-y^{2}} d y \\
& =\left(b^{*}\right)^{-1 / 2}\left(\frac{e^{-y_{0}^{2}}}{2 y_{0}}-\int_{y_{0}}^{\infty} \frac{e^{-y^{2}}}{2 y^{2}}\right)
\end{aligned}
$$

with $y_{0}=\left(b^{*}\right)^{1 / 2}(k-1)$. Thus in view of $(3.8)$ we deduce that

$$
\begin{aligned}
\sum_{p=k}^{\infty} e^{-b^{*} p^{2}}=O\left(\frac{e^{-y_{0}^{2}}}{\left(b^{*}\right)^{1 / 2} y_{0}}\right) & =O\left(\frac{\exp \left[\frac{1}{2} \omega(1-\epsilon) \log b(N)\right]}{b(N)^{1 / 2}[\log (1 / b(N))]^{1 / 2}}\right) \\
& =O\left(b(N)^{(1 / 2) \omega_{1}-(1 / 2)}\right)
\end{aligned}
$$

since $\omega_{1}<\omega$ and $\epsilon$ may be chosen small enough. Hence (3.8) follows from (3.14). 


\section{$\S 4$. Behaviors of differences}

LEMma 4.1. Let $P(z)$ be a polynomial satisfying $\operatorname{deg}[P(z)]=m$, and $|P(z)| \leq M$ for $|z| \leq r$. Then, if $R \geq r$, we have

$$
|\Delta P(z)| \leq \frac{e M m(R+1)^{m-1}}{r^{m}}, \quad|z| \leq R .
$$

Proof. By [5] p.337 Lemma 7, we have $\left|P^{\prime}(z)\right| \leq e M m(R+1)^{m-1} / r^{m}$, $|z| \leq R+1$. Hence

$$
|\Delta P(z)|=|P(z+1)-P(z)| \leq \int_{0}^{1}\left|P^{\prime}(z+t)\right| d t \leq \frac{e M m(R+1)^{m-1}}{r^{m}},
$$

since $|z+t| \leq R+1$ if $|z| \leq R$.

Theorem 4.2. Let $f(z)$ be as in (2.5), and suppose that $r \notin E$. Then

$$
\left(\frac{z}{N}\right)^{h} \Delta^{h} f(z)=f(z)+O\left(\frac{k}{N}\right) M(r, f), \quad|z|=r,
$$

for $h \in \mathbb{N}$, with $N=\nu^{*}(r)$ and $k$ in (3.8).

Proof. We write

$$
f(z)=\sum_{n=N-k}^{N+k} a_{n} z(n)+\phi(z)=z(N-k) P(z)+\phi(z) .
$$

First we treat $\phi(z)$. By Theorem 3.3,

$$
\begin{aligned}
r^{h}\left|\Delta^{h} \phi(z)\right| & =r^{h}\left|\sum_{|n-N|>k} a_{n} n(n-1) \cdots(n-h+1) z(n-h)\right| \\
& \leq \sum_{|n-N| \geq k} n^{h}\left|a_{n}\right| r^{*(n)} \frac{r^{h}}{(r+n-h) \cdots(r+n-1)} \\
& =o\left(\mu^{*}(r) N^{h} b(N)^{(1 / 2) \omega_{1}-(1 / 2)}\right),
\end{aligned}
$$

if $h \leq n$. We choose $\omega=10$ and $\omega_{1}=9$ in the statements of Theorem 3.3. Then we have $b(N)^{(1 / 2) \omega_{1}-(1 / 2)}=o\left(N^{-4}\right)$ so that

$$
r^{h}\left|\Delta^{h} \phi(z)\right|=o\left(\mu^{*}(r) N^{h-4}\right) .
$$


Secondly, we estimate $P(z)$ and $\Delta^{\ell} P(z)$ below. We remark that $k=$ $k(r)$ in (3.8) is smaller than $r$, if $r$ is sufficiently large, since $N(r)^{\gamma}<r$ with $\gamma>2$. Thus we may assume that $N-k$ is a positive integer. We see that

$$
\sum_{j=1}^{N-k-1} \frac{j}{r}<\frac{(N-k)(N-k-1)}{N^{\gamma}}<1
$$

For $0<x_{j}<1, j=1, \ldots, n, \sum_{j=1}^{n} x_{j}<1$, we have $\prod_{j=1}^{n}\left(1-\frac{x_{j}}{2}\right) \geq$ $1-\sum_{j=1}^{n} x_{j}$. Hence we get

$$
\begin{aligned}
|z(N-k)| & =|z(z-1) \cdots(z-(N-k)+1)| \\
& \geq r(r-1) \cdots(r-(N-k-1)) \\
& \geq r^{N-k}\left(1-\frac{1}{r}\right) \cdots\left(1-\frac{N-k-1}{r}\right) \\
& \geq\left(1-2 \sum_{j=1}^{N-k-1} \frac{j}{r}\right) r^{N-k} \\
& \geq r^{N-k}\left(1-\frac{(N-k)(N-k-1)}{N^{\gamma}}\right) \\
& =r^{N-k}(1-\epsilon), \quad \epsilon \rightarrow 0 \text { as } r \rightarrow \infty .
\end{aligned}
$$

By (2.21) in Remark 2.2,

$$
\begin{aligned}
|P(z)| & =|z(N-k)|^{-1}\left|\sum_{|n-N| \leq k} a_{n} z(n)\right| \leq r^{k-N}(1+\epsilon) \sum_{|n-N| \leq k}\left|a_{n}\right| r^{*(n)} \\
& \leq 2 k\left(1+\epsilon^{\prime}\right) r^{k-N} M(r, f)
\end{aligned}
$$

where $\epsilon^{\prime}=(1+\epsilon)\left(1+\max _{|n-N| \leq k} \epsilon_{n}\right)-1$. By definitions of $\epsilon, \epsilon_{n}$ and $k$, we see that $\epsilon^{\prime} \rightarrow 0$ as $r \rightarrow \infty$. Set $M_{0}=2 k\left(1+\epsilon^{\prime}\right) r^{k-N} M(r, f)$. An application of Lemma 4.1, with $R=r$ and $P(z)$ in (4.2), shows that

$$
|\Delta P(z)| \leq \frac{2 e M_{0} k(r+1)^{2 k-1}}{r^{2 k}} \leq O\left(\frac{k}{r}\right) M_{0} .
$$

Namely, we have $|\Delta P(z)| \leq C_{1}(k / r) M_{0}$ for a constant $C_{1}$. Similarly we get

$$
\left|\Delta^{2} P(z)\right|=|\Delta(\Delta P(z))| \leq \frac{2 k e C_{1} M_{0}(k / r)(r+1)^{2 k-2}}{r^{2 k-1}} \leq O\left(\frac{k}{r}\right)^{2} M_{0} .
$$


Repeating this process, we obtain for any positive integer $\ell$

$$
\left|\Delta^{\ell} P(z)\right|=O\left(\frac{k}{r}\right)^{\ell} M_{0}
$$

By (2.4),

$$
\begin{aligned}
\Delta^{h}(z(N- & k) P(z)) \\
= & \frac{(N-k) !}{(N-k-h) !} z(N-k-h) P(z) \\
& +\sum_{p=1}^{h}\left(\begin{array}{l}
h \\
p
\end{array}\right) \Delta^{h-p} z(N-k) \Delta^{p} P(z) \\
& +\sum_{\ell=1}^{h}\left(\begin{array}{c}
h \\
\ell
\end{array}\right) \sum_{p=0}^{h-\ell}\left(\begin{array}{c}
h-\ell \\
p
\end{array}\right) \Delta^{h-p} z(N-k) \Delta^{p+\ell} P(z) .
\end{aligned}
$$

We estimate the right-hand side of (4.5). To do this, we note that

$$
\begin{aligned}
\Delta^{h-p} z(N-k) & =\frac{(N-k) !}{(N-k-h+p) !} z(N-k-h+p) \\
& =O\left(\frac{(N-k) !}{(N-k-h) !}\left(1+\frac{r}{N}\right)^{p} z(N-k-h)\right) \\
& =O\left(\frac{(N-k) !}{(N-k-h) !} z(N-k-h)\right) .
\end{aligned}
$$

Thus, in view of (4.2), (4.3) and (4.4),

(4.5) $\quad \Delta^{h}(z(N-k) P(z))$

$$
\begin{aligned}
& =\frac{(N-k) !}{(N-k-h) !} z(N-k-h)\left(P(z)+O\left(\frac{k}{r}\right) M_{0}\right) \\
& =\frac{(N-k) !}{(N-k-h) !} \frac{z(N-k-h)}{z(N-k)}\left(f(z)+O\left(\mu^{*}(r) N^{-4}\right)+O\left(r^{N-k} M_{0} \frac{k}{r}\right)\right) .
\end{aligned}
$$

We suppose $\chi^{*}=\chi\left(f^{*}\right)<1 / 2$, where $f^{*}(z)$ is the function given by (2.11), corresponding to $f(z)$ of (2.5). Then we can take $r>N^{\gamma}, \gamma=$ $1 /\left(\chi^{*}+\epsilon\right)>2$. Hence by $(2.21)$

$$
\mu^{*}(r) N^{-4} \leq\left(1+\epsilon_{N}\right) M(r, f) N^{-4}=O\left(\frac{k}{r}\right) M(r, f) .
$$


Also $r^{N-k} M_{0}=2 k\left(1+\epsilon^{\prime}\right) M(r, f)$. Thus combining (4.2), (4.3), (4.6), we deduce

$$
\begin{aligned}
z^{h} \Delta^{h} f(z) & =\frac{(N-k) !}{(N-k-h) !}\left(f(z)+O\left(\frac{k}{N}\right) M(r, f)\right) \\
& =N^{h}\left(f(z)+O\left(\frac{k}{N}\right) M(r, f)\right) \quad(|z|=r) .
\end{aligned}
$$

\section{§5. Proof of Theorem 1.1}

Let $f(z)$ be an transcendental entire solution of the difference equation (1.1). We can apply (4.1) to (1.1). Then for $*$-normal $z,|z|=r \notin E$ and $|f(z)|=M(|z|, f)$, we obtain

$$
\sum_{j=0}^{p} a_{A_{j}}^{(j)} N(r)^{j} z^{A_{j}-j} f(z)(1+o(1))=0
$$

Thus we have

$$
N(r)=\nu^{*}(r)=L r^{\alpha}(1+o(1))
$$

for some $L$ and $\alpha$. The number $\alpha$ is determined as a slope of the Newton polygon for the equation (5.1), hence it is a rational number. By (2.19) $\alpha=\chi=\chi(f)$. Since

$$
\log \mu^{*}(r)=\log \left|a_{N}\right|+\log r+\log (r+1)+\cdots+\log (r+N-1)
$$

with $N=\nu^{*}(r)$, we have

$$
\log \mu^{*}(r)=\log \left|a_{N}\right|+N \log r+\sum_{k=1}^{N-1} \log \left(1+\frac{k}{r}\right)
$$

Let $r_{1}<r_{2}<\ldots$ be such that $\nu^{*}(r)=N_{j}$ for $r_{j} \leq r<r_{j+1}$. Note that

$$
\frac{d}{d r} \log \mu^{*}(r)=\frac{\nu^{*}(r)}{r}+\frac{d}{d r} \sum_{k=1}^{N_{j}-1} \log \left(1+\frac{k}{r}\right), \quad \text { for } r_{j} \leq r<r_{j+1}
$$

and that

$$
\log \mu^{*}(r)-\log \mu^{*}\left(r_{j}\right)=\int_{r_{j}}^{r} \frac{\nu^{*}(t)}{t} d t+\sum_{k=1}^{N_{j}-1} \log \left(1+\frac{k}{r}\right)-\sum_{k=1}^{N_{j}-1} \log \left(1+\frac{k}{r_{j}}\right) .
$$


Thus for $r_{j+1} \leq r<r_{j+2}$,

$$
\begin{aligned}
& \log \mu^{*}(r)=\log \mu^{*}\left(r_{j+1}\right)+\int_{r_{j+1}}^{r} \frac{\nu^{*}(t)}{t} d t+\sum_{k=1}^{N_{j+1}-1} \log \left(1+\frac{k}{r}\right) \\
& -\sum_{k=1}^{N_{j+1}-1} \log \left(1+\frac{k}{r_{j+1}}\right) \\
& =\log \mu^{*}\left(r_{j}\right)+\int_{r_{j}}^{r_{j+1}} \frac{\nu^{*}(t)}{t} d t+\int_{r_{j+1}}^{r} \frac{\nu^{*}(t)}{t} d t \\
& +\sum_{k=1}^{N_{j}-1} \log \left(1+\frac{k}{r_{j+1}}\right)-\sum_{k=1}^{N_{j}-1} \log \left(1+\frac{k}{r_{j}}\right) \\
& +\sum_{k=1}^{N_{j+1}-1} \log \left(1+\frac{k}{r}\right)-\sum_{k=1}^{N_{j+1}-1} \log \left(1+\frac{k}{r_{j+1}}\right) \\
& =\log \mu^{*}\left(r_{j}\right)+\int_{r_{j}}^{r} \frac{\nu^{*}(t)}{t} d t+\sum_{k=1}^{N_{j+1}-1} \log \left(1+\frac{k}{r}\right) \\
& -\sum_{k=N_{j}}^{N_{j+1}-1} \log \left(1+\frac{k}{r_{j+1}}\right)-\sum_{k=1}^{N_{j}-1} \log \left(1+\frac{k}{r_{j}}\right) \\
& =\log \mu^{*}\left(r_{1}\right)+\int_{r_{1}}^{r} \frac{\nu^{*}(t)}{t} d t+\sum_{k=1}^{N_{j+1}-1} \log \left(1+\frac{k}{r}\right) \\
& -\sum_{\ell=1}^{j+1} \sum_{k=N_{\ell-1}}^{N_{\ell}-1} \log \left(1+\frac{k}{r_{\ell}}\right)
\end{aligned}
$$

with $N_{0}=1$. We write the last sum as $R_{N}$ with $N=N_{j+1}$.

$$
\sum_{k=N_{\ell-1}}^{N_{\ell}-1} \log \left(1+\frac{k}{r_{\ell}}\right) \leq \sum_{k=1}^{N_{\ell}-1} \log \left(1+\frac{k}{r_{\ell}}\right) \leq \frac{1}{N_{\ell}^{\gamma-2}}
$$

Since $N_{\ell} \geq \ell$, we have

$$
R_{N} \leq \sum_{\ell=1}^{j+1} \frac{1}{\ell \gamma-2}
$$


When $\gamma \geq 3$, obviously we have $R_{N}=O(\log N)=o\left(r^{\chi}\right)$. When $2<\gamma<3$, we have

$$
R_{N} \leq 1+\int_{1}^{N} t^{2-\gamma} d t \leq 1+\frac{N^{3-\gamma}}{3-\gamma}
$$

Since $N \leq r^{1 / \gamma}$, we get $R_{N}=O\left(r^{(3-\gamma) / \gamma)}\right)=O\left(r^{(3 / \gamma-1)}\right)=O\left(r^{(3 \chi+3 \varepsilon-1)}\right)=$ $r^{\chi} O\left(r^{2 \chi-1+3 \varepsilon}\right)$. If $0<\varepsilon<(1-2 \chi) / 3$, we obtain $R_{N}=o\left(r^{\chi}\right)$. Furthermore,

$$
\sum_{k=1}^{N_{j+1}-1} \log \left(1+\frac{k}{r}\right)=O\left(N^{2-\gamma}\right)
$$

Hence we obtain

$$
\begin{aligned}
\log \mu^{*}(r)= & \log \mu^{*}\left(r_{1}\right)+\int_{r_{1}}^{r} \frac{\nu^{*}(t)}{t} d t+\sum_{k=1}^{N_{j+1}-1} \log \left(1+\frac{k}{r}\right) \\
& -\sum_{\ell=1}^{j+1}\left(\sum_{k=N_{\ell-1}}^{N_{\ell}-1} \log \left(1+\frac{k}{r_{\ell}}\right)\right)=\frac{L}{\chi} r^{\chi}(1+o(1)) .
\end{aligned}
$$

We note that by the definition of $*$-nomal and by the similar arguments in [5, pp. 330-334], we have $M(r, f) \leq \mu^{*}(r)\left(\log \mu^{*}(r)\right)^{1 / 2+\delta^{\prime}}$ for a positive constant $\delta^{\prime}$. Combining this and (2.21), we obtain $\mu^{*}(r) \leq K_{\nu^{*}(r)} M(r, f) \leq$ $\mu^{*}(r)\left(\log \mu^{*}(r)\right)^{1 / 2+\delta}$, for a positive constant $\delta$. Hence we conclude that

$$
\log M(r, f)=\frac{L}{\chi} r^{\chi}(1+o(1)) .
$$

By the same arguments as in [6, pp. 259-261], we can show that (5.5) holds for $r \in \mathbb{R}_{+}$, without exceptions.

\section{$\S 6 . \quad$ Examples}

In this section we give examples of difference equations which have entire solutions of order less than $1 / 2$. We start with a remark.

Remark 6.1. If $f(z)$ is a meromorphic solution of a difference equation with a pole coinciding with neither of the zeros of $a_{j}(z)$, then $N(r, f) \geq$ $\operatorname{Lr}(L>0)$, hence it is of order not less than 1 . 
Remark 6.2. As we said above, we want to find difference equations of the form (1.1) which have entire solutions of order less than $1 / 2$. We can easily see that the order $p$ is at least 3 by Theorem 1.1 and the Newton polygon. Let us show that there exists actually such a difference equation of order $p=3$.

Remark 6.3. Consider the following equation of third order, with some constants $b_{j}$ and $c_{0}$,

$$
\begin{aligned}
b_{3} z(z-1)(z-2) \Delta^{3} f(z-3) & +b_{2} z(z-1) \Delta^{2} f(z-2) \\
& +b_{1} z \Delta f(z-1)+\left(c_{0} z+b_{0}\right) f(z)=0
\end{aligned}
$$

For later use we write $b_{3}(z)=b_{3} z(z-1)(z-2), b_{2}(z)=b_{2} z(z-1), b_{1}(z)=$ $b_{1} z$. Put a formal solution in the form

$$
f(z)=\sum_{n=0}^{\infty} \alpha_{n} z_{\lambda}(n), \quad z_{\lambda}(n)=\frac{\Gamma(z+1)}{\Gamma(z+1-\lambda-n)}, \quad \alpha_{0} \neq 0
$$

When $\lambda \in \mathbb{N} \cup\{0\},(6.2)$ will give an entire solution if the right hand side converges.

Note that $\Delta^{3} f(z-3)=\sum_{n=0}^{\infty} \alpha_{n} \Delta^{3}(z-3)_{\lambda}(n)$. For each term, we have

$$
\begin{aligned}
z(z-1) & (z-2) \Delta^{3}(z-3)_{\lambda}(n) \\
& =z(z-1)(z-2) \Delta^{2}\left\{\frac{\Gamma(z-1)}{\Gamma(z-1-\lambda-n)}-\frac{\Gamma(z-2)}{\Gamma(z-2-\lambda-n)}\right\} \\
& =z(z-1)(z-2) \Delta^{2}\left\{\frac{(\lambda+n) \Gamma(z-2)}{\Gamma(z-1-\lambda-n)}\right\} \\
& =(\lambda+n) z(z-1)(z-2) \Delta\left\{\frac{\Gamma(z-1)}{\Gamma(z-\lambda-n)}-\frac{\Gamma(z-2)}{\Gamma(z-1-\lambda-n)}\right\} \\
& =(\lambda+n) z(z-1)(z-2) \Delta\left\{\frac{(\lambda+n-1) \Gamma(z-2)}{\Gamma(z-\lambda-n)}\right\} \\
& =(\lambda+n)(\lambda+n-1)(\lambda+n-2) \frac{z(z-1)(z-2) \Gamma(z-2)}{\Gamma(z+1-\lambda-n)} \\
& =(\lambda+n)(\lambda+n-1)(\lambda+n-2) \frac{\Gamma(z+1)}{\Gamma(z+1-\lambda-n)} \\
& =(\lambda+n)(\lambda+n-1)(\lambda+n-2) z_{\lambda}(n) .
\end{aligned}
$$


Similarly

$$
\begin{aligned}
z(z-1) \Delta^{2} f(z-2) & =\sum_{n=0}^{\infty}(\lambda+n)(\lambda+n-1) \alpha_{n} z_{\lambda}(n) \\
z \Delta f(z-1) & =\sum_{n=0}^{\infty}(\lambda+n) \alpha_{n} z_{\lambda}(n)
\end{aligned}
$$

Further noting that $z f(z)=\sum_{n=0}^{\infty} \alpha_{n} z \cdot z_{\lambda}(n)$, and that

$$
\begin{aligned}
z \cdot z_{\lambda}(n) & =\frac{(z-\lambda-n) \Gamma(z+1)}{(z-\lambda-n) \Gamma(z-\lambda-n)}+\frac{(\lambda+n) \Gamma(z+1)}{\Gamma(z+1-\lambda-n)} \\
& =z_{\lambda}(n+1)+(\lambda+n) z_{\lambda}(n),
\end{aligned}
$$

we get

$$
\begin{aligned}
& \sum_{n=0}^{\infty}(\lambda+n)(\lambda+n-1)(\lambda+n-2) b_{3} \alpha_{n} z_{\lambda}(n) \\
& \quad+\sum_{n=0}^{\infty}(\lambda+n)(\lambda+n-1) b_{2} \alpha_{n} z_{\lambda}(n)+\sum_{n=0}^{\infty}(\lambda+n) b_{1} \alpha_{n} z_{\lambda}(n) \\
& +\left(\lambda c_{0}+b_{0}\right) \alpha_{0} z_{\lambda}(0)+\sum_{n=1}^{\infty}\left[c_{0} \alpha_{n-1}+\left((\lambda+n) c_{0}+b_{0}\right) \alpha_{n}\right] z_{\lambda}(n)=0 .
\end{aligned}
$$

Thus we obtain the indicial equation for (6.1)

$$
\lambda(\lambda-1)(\lambda-2) b_{3}+\lambda(\lambda-1) b_{2}+\lambda\left(b_{1}+c_{0}\right)+b_{0}=0
$$

When roots of (6.4) differ by integers, we get some trouble in determining coefficients in (6.2). We take so that $b_{0}=0, c_{0}=-1, b_{1}=2\left(b_{1}+c_{0}=1\right)$ and $b_{2}=1, b_{3}=6$, then $\lambda=0,4 / 3,3 / 2$. We remark that when $\lambda=0$, $z_{\lambda}(n)=z_{0}(n)$ coincides with $z(n)$. Then

$$
\begin{aligned}
\sum_{n=1}^{\infty} 6 n(n-1)(n-2) & \alpha_{n} z(n)+\sum_{n=1}^{\infty} n(n-1) \alpha_{n} z(n) \\
& +\sum_{n=1}^{\infty} 2 n \alpha_{n} z(n)-\sum_{n=1}^{\infty}\left[\alpha_{n-1}+n \alpha_{n}\right] z(n)=0
\end{aligned}
$$


from which we have

$$
\begin{aligned}
& n(2 n-3)(3 n-4) \alpha_{n}=\alpha_{n-1}, \\
& \alpha_{n}=\frac{1}{6} \frac{1}{n\left(n-\frac{3}{2}\right)\left(n-\frac{4}{3}\right)} \alpha_{n-1}=\frac{1}{6} \frac{1}{n^{3}} \frac{1}{(1-(3 / 2 n))(1-(4 / 3 n))} \alpha_{n-1} .
\end{aligned}
$$

Thus we get

$$
\alpha_{n}=\left(\frac{1}{6}\right)^{n} \frac{1}{(n !)^{3}}\left\{\prod_{t=1}^{n}\left(1-\frac{3}{2 t}\right)\left(1-\frac{4}{3 t}\right)\right\}^{-1} \alpha_{0} .
$$

We have

$$
\left|\prod_{t=1}^{n}\left(1-\frac{3}{2 t}\right)^{-1}\left(1-\frac{4}{3 t}\right)^{-1}\right|=O\left(\exp \left(C_{0} \sum_{t=1}^{n} \frac{1}{t}\right)\right)=O\left(n^{C_{0}}\right)
$$

for some $C_{0}$. Thus we see that $\alpha_{k}=O\left(6^{-k}(k !)^{-3} k^{C_{0}}\right)$, and hence

$$
\begin{aligned}
\left|\beta_{n}\right| & \leq O\left(\sum_{k=n}^{\infty} \alpha_{k}\left|\eta_{n}^{(k)}\right|\right)=O\left(\sum_{k=n}^{\infty}\left(\frac{1}{6}\right)^{k} \frac{k^{C_{0}}}{(k !)^{3}}\left(\frac{(k-1) !}{(n-1) !}\right)^{2} \frac{1}{(k-n) !}\right) \\
& =O\left(\frac{1}{(n !)^{2}} \sum_{k=n}^{\infty} \frac{1}{k !(k-n) !}\right)=O\left(\frac{1}{(n !)^{3}}\right),
\end{aligned}
$$

which shows that $\left|\beta_{n}\right|=O\left(e^{3 n} n^{-3 n}\right)$. Hence we obtain $\chi(f) \leq 1 / 3$. Using the relations

$$
\begin{aligned}
& b_{3}^{*}(z)=b_{3}(z)+b_{2}(z)+b_{1}(z)+\left(c_{0} z+b_{0}\right), \\
& b_{2}^{*}(z)=b_{2}(z)+2 b_{1}(z)+3\left(c_{0} z+b_{0}\right) \\
& b_{1}^{*}(z)=b_{1}(z)+3\left(c_{0} z+b_{0}\right)
\end{aligned}
$$

the equation (6.1) is written as

$$
\begin{aligned}
b_{3}^{*}(z) \Delta^{3} f(z-3)+b_{2}^{*}(z) \Delta^{2} f(z-3)+b_{1}^{*}(z) \Delta & f(z-3) \\
& +\left(c_{0} z+b_{0}\right) f(z-3)=0 .
\end{aligned}
$$

In particular, we see that $f(z)$ satisfies the following equation in our special case, namely $b_{0}=0, c_{0}=-1, b_{1}=2, b_{2}=1$ and $b_{3}=6$,

$$
\left(6 z^{2}+19 z+15\right) \Delta^{3} f(z)+(z+3) \Delta^{2} f(z)-\Delta f(z)-f(z)=0 .
$$

Hence the slope of the Newton polygon is $1 / 3$, and by Theorem 1.1 we have $\log M(r, f)=L r^{1 / 3}(1+o(1))$. 
EXAMPLE 6.1. Here we give a difference equation which has two solutions $f$ and $g$ of different order $\chi(f) \neq \chi(g), \chi(f), \chi(g)<1 / 2$. First consider the difference equation

$$
\begin{aligned}
& \begin{aligned}
& L_{1}[f(z)]= b_{3}(z) \Delta^{3} f(z-3)+b_{2}(z) \Delta^{2} f(z-2) \\
&+b_{1}(z) \Delta f(z-1)+b_{0}(z) f(z)=0, \\
& b_{3}(z)=6 z(z-1)(z-2), b_{2}(z)=z(z-1), b_{1}(z)=2 z, b_{0}(z)=-z,
\end{aligned}
\end{aligned}
$$

which also can be written as

$$
\begin{aligned}
L_{2}[f(z)]= & \left(6 z^{3}+19 z^{2}+15 z\right) \Delta^{3} f(z)+\left(z^{2}+3 z\right) \Delta^{2} f(z)-z \Delta f(z)-z f(z) \\
& =b_{3}^{*}(z) \Delta^{3} f(z)+b_{2}^{*}(z) \Delta^{2} f(z)+b_{1}^{*}(z) \Delta f(z)+b_{0}^{*}(z) f(z)=0 .
\end{aligned}
$$

Its solution $f(z)=\sum_{n=0}^{\infty} \alpha_{n} z(n), z(n)=z(z-1) \cdots(z-n+1)$, satisfies

$$
n(2 n-3)(3 n-4) \alpha_{n}=\alpha_{n-1} .
$$

Put $g(z)=z f(z)$. Then $g(z)$ satisfies an equation of the form

$$
L_{3}[g(z)]=b_{3}^{* *}(z) \Delta^{3} g(z)+b_{2}^{* *} \Delta^{2} g(z)+b_{1}^{* *} \Delta g(z)+b_{0}^{* *}(z) g(z)=0 .
$$

Next we consider another difference equation

$$
\begin{aligned}
L_{5}[h(z)] & =\sum_{j=0}^{5} c_{j}^{*}(z) \Delta^{j} h(z-j)=0, \\
c_{j}^{*}(z) & =c_{j} \cdot z(z-1) \cdots(z-j+1) \quad(1 \leq j \leq 5), \\
c_{5} & =36, c_{4}=228, c_{3}=271, c_{2}=28, c_{1}=3, \quad c_{0}^{*}(z)=c_{0} z, c_{0}=-1 .
\end{aligned}
$$

Note that $c_{1}+c_{0}=2$. The equation $L_{5}[h(z)]=0$ can be also written as

$$
L_{5}[h(z)]=c_{5}(z) \Delta^{5} h(z)+\cdots+c_{1}(z) \Delta h(z)+c_{0}(z) h(z)=0 .
$$

Its solution $h(z)=\sum_{n=0}^{\infty} \gamma_{n} z(n)$ satisfies

$$
n(2 n-1)(2 n-3)(3 n-1)(3 n-4) \gamma_{n}=\gamma_{n-1}, \quad n \geq 1,
$$

from which we can show that $\chi(h) \leq 1 / 5$, and our theorem shows that $\chi(h)=1 / 5$, since the slope of the Newton polygon for $L_{5}[h]$ is $1 / 5$. Now put $L^{*}[f(z)]=L_{3}\left[L_{5}[f(z)]\right]$. Above $h(z)$ is an entire solution of $L^{*}[f(z)]=0$ which is of order $1 / 5$. Another entire solution $d(z)=\sum_{n=0}^{\infty} \delta_{n} z(n)$ is given 
by $L_{5}[d(z)]=g(z)$, where $g(z)=z f(z)$ is the solution of $L_{3}[g(z)]=0$, which was obtained above. Therefore we have

$$
n(2 n-1)(2 n-3)(3 n-1)(3 n-4) \delta_{n}-\delta_{n-1}=\alpha_{n-1}+n \alpha_{n}
$$

We can write $\delta_{n}=\delta_{n}^{(1)}+\delta_{n}^{(2)}$, where

$$
\begin{aligned}
& n(2 n-1)(2 n-3)(3 n-1)(3 n-4) \delta_{n}^{(1)}-\delta_{n-1}^{(1)}=\alpha_{n-1} \\
& n(2 n-1)(2 n-3)(3 n-1)(3 n-4) \delta_{n}^{(2)}-\delta_{n-1}^{(2)}=n \alpha_{n} .
\end{aligned}
$$

Let $\delta_{n}^{(1)} / \alpha_{n}=D_{n}$. Then we get

$$
(2 n-1)(3 n-1) D_{n}=1+D_{n-1} .
$$

Put $(2 n-1)(3 n-1) D_{n}=D_{n}^{\prime}$. Then

$$
\begin{aligned}
& D_{n}^{\prime}=1+\frac{1}{(2 n-3)(3 n-4)} D_{n-1}^{\prime} \\
= & 1+\frac{1}{(2 n-3)(3 n-4)}+\frac{1}{(2 n-3)(3 n-4)(2 n-5)(3 n-7)} D_{n-2}^{\prime}=\cdots,
\end{aligned}
$$

hence we know that $\delta_{n}^{(1)} / \alpha_{n}=O\left(n^{-2}\right), \alpha_{n} / \delta_{n}^{(1)}=O\left(n^{2}\right) . \delta_{n}^{(2)}$ can also be estimated. Since they are non-negative, we see that $d(z)$ is of the same order as $g(z)$, which is of the same order as $f(z)$. Thus we get

$$
\chi(h)=1 / 5, \quad \chi(d)=1 / 3 .
$$

Finally we will consider slopes of the Newton polygon for

$$
L^{*}[f(z)]=\sum_{j=0}^{8} b_{j}^{* * *}(z) \Delta^{j} f(z)=0 .
$$

A direct calculation yields $\operatorname{deg}\left[b_{8}^{* * *}\right]=11, \operatorname{deg}\left[b_{7}^{* * *}\right] \leq 10, \operatorname{deg}\left[b_{6}^{* * *}\right] \leq 9$, $\operatorname{deg}\left[b_{5}^{* * *}\right]=9, \operatorname{deg}\left[b_{4}^{* * *}\right]=8, \operatorname{deg}\left[b_{3}^{* * *}\right]=7, \operatorname{deg}\left[b_{2}^{* * *}\right]=6, \operatorname{deg}\left[b_{1}^{* * *}\right]=5$ and $\operatorname{deg}\left[b_{0}^{* * *}\right]=5$. Therefore the Newton polygon is the convex hull of the union of

$$
D=\{(x, y) \mid x \geq 0, y \leq 0\}, \quad(3,4)+D, \quad(8,5)+D
$$

and its slopes are $1 / 3$ and $1 / 5$. 


\section{REFERENCES}

[1] S. B. Bank and R. P. Kaufman, An extension of Hölder's theorem concerning the Gamma function, Funkcialaj Ekvacioj, 19 (1976), 53-63.

[2] W. Bergweiler, K. Ishizaki, and N. Yanagihara, Growth of meromorphic solutions of some functional equations I, Aequationes Math., 63 (2002), 140-151.

[3] R. P. Boas, Jr., Entire functions, Academic Press Inc., New York, 1954.

[4] G. Gundersen, G. Steinbart, M. Enid and S. Wang, The possible orders of solutions of linear differential equations with polynomial coefficients, Trans. Amer. Math. Soc., 350 (1998), 1225-1247.

[5] W. K. Hayman, The local growth of power series: A survey of the Wiman-Valiron method, Canad. Math. Bull., 17 (1974), 317-358.

[6] W. Helmrath and J. Nikolaus, Ein elementarer Beweis bei der Anwendung der Zentralindexmethode auf Differentialgleichungen, Complex Variables Theory Appl., 3 (1984), 253-262.

[7] Kövari, T., On the Borel exceptional values of lacunary integral functions, J. Analyse Math., 9 (1961), 71-109.

[8] Laine, I., Nevanlinna theory and complex differential equations, W. Gruyter, Berlin-New York, 1992.

[9] Nörlund, N.E., Vorlesungen über Differenzenrechnung, Chelsea Publ., New York, 1954.

[10] Wittich, H., Neuere Untersuchungen über eindeutige analytische Funktionen, Springer-Verlag, 1955.

K. Ishizaki

Department of Mathematics

Nippon Institute of Technology

4-1 Gakuendai Miyashiro, Minamisaitama

Saitama-ken, 345-0826

Japan

ishi@nit.ac.jp

N. Yanagihara

Minami-Iwasaki 671-18, Ichihara-City

Chiba-ken 290-0244

Japan

yanagihara-nm@icntv.ne.jp 